\title{
El derecho del mar y el cambio climático en el Ártico: ¿estrechos internacionales o protección y preservación del medio marino?
}

Fecha de recepción: 12 de enero de 2018

Fecha de aceptación: $1^{\circ}$ de agosto de 2018

Doi: dx.doi.org/10.12804/revistas.urosario.edu.co/acdi/a.7567

\section{Leopoldo M. A. Godio*}

Resumen: el presente trabajo analiza la situación del océano Ártico y los efectos del cambio climático aplicados al paso del Noroeste, una vía de navegación que une el Pacífico y el Atlántico; es objeto de una importante controversia internacional entre Estados Unidos y Canadá; y además ha llamado la atención de Estados como Japón, Noruega, China e, incluso, la Unión Europea.

* Abogado y magíster en Relaciones Internacionales (UBA). Profesor de Derecho Internacional Público, UBA y UCA; miembro titular de la AADI; miembro del Instituto de Derecho Internacional del CARI, investigador adscripto del Instituto de Investigaciones Jurídicas y Sociales Ambrosio L. Gioja; director del Proyecto DeCyT (DCT1615) "La contribución del sistema de solución de controversias de la Convemar a la consolidación del derecho del mar" (Facultad de Derecho, UBA); y miembro del Instituto de Derecho Internacional de la Academia Nacional de Derecho y Ciencias Sociales de Buenos Aires, entre otros. Correo electrónico: leopoldogodio@derecho.uba.ar

Para citar este artículo: Godio, L. M., "El derecho del mar y el cambio climático en el Ártico: ¿estrechos internacionales o protección y preservación del medio marino?", Anuario Colombiano de Derecho Internacional (ACDI), 2019, 12, pp. 19-42. Doi: dx.doi.org/10.12804/revistas.urosario.edu.co/acdi/a.7567 
Este escenario plantea la evidente necesidad de comprender no solo su particular situación frente al efecto del cambio climático, sino también el desafío de su protección y preservación ante el potencial que presenta para el comercio marítimo internacional.

Palabras clave: cambio climático, navegación, buques, estrechos internacionales, medio marino.

Law of the Sea and Climate Change: ¿International Straits or Protection and Preservation of the Marine Environment?

Abstract: This work analyzes the situation of the Arctic Ocean and the effects of climate change applied to the Northwest Passage, a waterway linking the Pacific and the Atlantic, and is the subject of a relevant international dispute between the United States and Canada; which also has attracted the interest of States such as Japan, Norway, China and even the European Union.

This scenario raises the obvious need to understand not only the particular situation facing the impact of climate change, but also the challenge of protecting and preserving them against the potential offered for international maritime trade.

Keywords: Climate change, navigation, vessels, international straits, marine environment.

O direito do mar e a mudança climática no Ártico: estreitos internacionais ou proteção e preservação do meio marino?

Resumo: o presente trabalho analisa a situação do oceano Ártico e os efeitos da mudança climática aplicados ao passo do Nordeste, uma via de navegação que une o Pacífico e o Atlântico; é objeto de uma importante controvérsia internacional entre os Estados Unidos e o Canadá; e, além disso tem chamado a atenção de Estados como o Japão, a Noruega, a China e, inclusive, a União Europeia.

Este cenário apresenta a evidente necessidade de compreender não só sua particular situação frente ao efeito da mudança climática, mas 
também o desafio de sua proteção e preservação ante o potencial que apresenta para o comércio marítimo internacional.

Palavras-chave: mudança climática, navegação, navios, estreitos internacionais, meio marino.

\section{Introducción}

El cambio climático se origina por las emisiones de gases de efecto invernadero y sus consecuencias se manifiestan, naturalmente, en un incremento de la temperatura promedio del planeta y el nivel de las precipitaciones, así como en el patrón y características que presentan los eventos naturales. ${ }^{1}$ Por otra parte, repercute ampliamente en distintos ámbitos de la actividad vinculada al bienestar de la población, como, por ejemplo, el comercio internacional y el establecimiento de barreras paraarancelarias, la producción de bienes y servicios relacionados con el ambiente, la acidificación de mares y océanos, la desaparición de territorios insulares y la afectación de la biodiversidad marina. ${ }^{2}$

Sin embargo, por razones de extensión, propondremos considerar solo un sector del planeta como parte de nuestro objeto de estudio, casualmente alejado de nuestra vida diaria: las áreas polares. ${ }^{3}$ Allí, las secuelas del

1 Cfr. Durán Cotrina, Patricia, "La economía del cambio climático", Revista Peruana de Derecho Internacional, setiembre-diciembre 2011, LXI, (144), pp. 179-196.

2 Respecto a las islas frente al aumento de las mareas y desastres naturales, ver González Napolitano, Silvina S., "Los desastres naturales y sus efectos en islas y Estados insulares: posibles soluciones jurídicas”, en González Napolitano, Silvina Sandra (dir.), Respuestas del derecho internacional a desastres y otras consecuencias de fenómenos naturales, SGN Editora, Avellaneda, 2015, pp. 97-110.

3 Albert Gore afirma que "los efectos más tremendos del cambio climático se están dando en el Ártico y la Antártida, en verdad más en el Ártico, ya que, a diferencia de la Antártida, el casquete de hielo del Ártico mide unos 3 metros, es decir, es más vulnerable a las temperaturas que se elevan rápidamente". Cfr. Gore, Albert, Una verdad incómoda: la crisis planetaria del calentamiento global y cómo afrontarla, Gedisa, Barcelona, 2007, pp. 126-129. Con más exactitud, Symonides asevera que el cambio climático y el aumento de la temperatura global conducen a una reducción de la capa de hielo que generó, desde el siglo Xxi, en el caso del Ártico, una disminución de entre 2 a 5 millones de $\mathrm{km}^{2}$, perdiendo así más del 50\% de su superficie, en el más grave de las hipótesis. Cfr. Symonides, Janusz, "Problems and controversies concerning freedom of navigation in the Arctic", en Del 
cambio climático presentan desafíos que van más allá de las actividades científicas, como, por ejemplo, el acceso a nuevos recursos vivos y no vivos de gran interés para la industria farmacéutica y energética. En ese sentido, recortando aún más la amplitud de temas posibles, en el presente trabajo optamos por analizar una de ellas: la aparición de vías navegables en el océano Ártico que, se intuye, adquirirán carácter permanente en pocos años y serán aptas para su utilización con fines de transporte marítimo comercial, uniendo el norte de los océanos Pacífico y Atlántico, tal como se cree que ocurrirá con el Northwest Passage. ${ }^{4}$

De este modo, se presenta como hipótesis de trabajo demostrar qué parte y sección de la Convención de las Naciones Unidas sobre el Derecho del Mar de 1982 (en adelante, la Convención, indistintamente) corresponde asignarle al Northwest Passage. ¿Se trata de una aplicación del régimen de "paso en tránsito" dispuesto en la sección 2 de la parte III, referida a los estrechos internacionales (y su eventual aplicación mutatis mutandis a las "aguas archipelágicas" previstas en la parte IV)? ¿Debe incluírsele como un sistema de "aguas interiores" comprendido en la sección 2 de la parte II o destinar las disposiciones de "zonas cubiertas de hielo", establecidas en la sección 8 de la parte XII, que regula la protección del medio marino? La respuesta a estas preguntas requiere dar contestación a tres aspectos clave: ¿debe priorizarse el medio marino respecto de la navegación? ¿Se trata de un espacio donde prevalece un uso comercial? ¿Puede tener el primer estatus para luego mutar, dinámicamente, al segundo?

\section{E1 cambio climático y las regiones polares: el océano Ártico}

El efecto del cambio climático posee efectos en el medio animal y vegetal, ya que las elevadas temperaturas alteran los ciclos atmosféricos y provocan fenómenos meteorológicos cuya frecuencia — e intensidad — es cada vez mayor. ${ }^{5}$ En las regiones polares, sus consecuencias resultan especialmente

Castillo, Lilian (ed.), Law of the sea, from Grotius to the International Tribunal for the Law of the Sea. Liber amicorum judge Hugo Caminos, Brill, Leiden, 2015, p. 225.

4 Ibid.

5 Cfr. Fernández Egea, Rosa M., "El cambio climático y la sostenibilidad de los recursos pesqueros del mar del Norte: la controversia comercial entre la Unión Europea y las islas Feroe", Revista del Instituto Español de Estudios Estratégicos (IEEE), 2014, 4, pp. 4-6. 
preocupantes al ignorarse la importancia e irreversibilidad del proceso de deshielo de los polos, el incremento del nivel del mar y la alteración de las corrientes marinas. ${ }^{6}$

Precisamente, en el Ártico la lista de perjuicios resulta extensa y afecta el precario balance entre agua y hielo, ${ }^{7}$ al punto de que el aumento, en una fracción de 1 grado centígrado en el promedio anual de la temperatura, puede transformar un extenso hielo oceánico en un gran mar abierto. ${ }^{8}$ En consecuencia, distintos Estados ribereños adoptaron medidas con el objeto de desarrollar nuevas técnicas sin afectar el ecosistema.?

Aunque la comunidad internacional no reconoce universalmente ningún reclamo que recaiga sobre el Ártico, ${ }^{10}$ este fue objeto de competencia entre los distintos Estados por la explotación de sus recursos, pero ninguno resultó exitoso. A pesar del atractivo que poseen sus riquezas para las grandes potencias, se trata de una zona con poca o nula injerencia de actividad humana. ${ }^{11}$ En ese orden de ideas, la disputa entre Estados Unidos y Canadá por el denominado Northwest Passage se encuentra inserta en este debate y su calificación como estrecho o aguas interiores, según el interés de cada uno de estos Estados, ${ }^{12}$ sin perjuicio de la posible cooperación

${ }^{6}$ Idem. En idéntico sentido, Martínez Vargas y Vega Barbosa señalan que el cambio climático y sus consecuencias en los mares y océanos representan una preocupación que excede los pronósticos realizados hace más de una década, especialmente en relación con la región del Ártico, además de alterar las líneas costeras, provocar inundaciones, afectar la habitalidad de territorios y hacer modificaciones en materia insular. Los autores ratifican la autoridad de la Convención de las Naciones Unidas sobre el Derecho del Mar de 1982 como principal instrumento vigente y coincidimos totalmente con su razonamiento. Cfr. Martínez Vargas, Juan R., Vega Barbosa, Giovanny et al., Tratado de Derecho del Mar, Tirant lo Blanch, Valencia, 2016, pp. 1101-1103.

7 Cfr. Byers, Michael, "Artic region: specific geographic issues", en Wolfrum, Rüdiger (ed.), The Max Planck encyclopedia of public international law, Oxford University Press, Oxford, 2012, para. 1 y ss.

8 Idem.

9 Idem.

10 Cfr. Duyck, Sebastien, "Drawing lessons for Artic governance from the Antartic Treaty System", en Alfredsson, Gudmundur, Koivurova, Timo \& Hossain, Kamrul (eds.), The yearbook of Polar law. Vol. 3, Brill, Leiden, 2011.

11 Idem.

12 Cfr. Byers, "Artic region...", op. cit., paras. 18-25. 
en el manejo y la administración de esta zona motivada, principalmente, por razones ambientales. ${ }^{13}$

\subsection{Implementación del principio precautorio}

Uno de los temas centrales es, sin duda, el principio 15 de la Declaración de Río, ${ }^{14}$ que describe a la emergencia climática como el más prominente - $\mathrm{y}$ controvertido- desarrollo del derecho internacional del medio ambiente en las últimas dos décadas, en particular, del principio precautorio. ${ }^{15} \mathrm{Lo}$ anterior no debe llamarnos la atención toda vez que el concepto resulta aplicable a las regiones polares como zonas cuyo detrimento, a causa principalmente de la variación y contaminación climática, es irreversible y su amenaza constante viene acompañada de potenciales y graves consecuencias.

Como ejercicio comparativo, vale la pena señalar que el Tratado Antártico contiene disposiciones indirectas, como los artículos V y IX, que regulan específicamente la adopción de medidas para evitar las actividades nucleares y radioactivas en la región; y la protección y conservación de los recursos vivos de la Antártida, respectivamente. Así mismo, su Protocolo de Protección del Medio Ambiente define a la Antártida, en el artículo $2^{\circ}$, como una "reserva natural, dirigida a la paz y la ciencia". Sin embargo, distintos proyectos destinados a la investigación pueden acelerar las consecuencias del cambio climático. Es por ello que debe considerarse si el principio precautorio no solo corresponde ser aplicado en lo que respecta

13 Incluso puede sostenerse que, como reflejo de esa concientización, se cristalizó la adopción del Tratado Antártico, seguido de acuerdos sectoriales como: Convention on the Conservation of Antartic Seals, 1972, 11 ILM 251; Convention on the Conservation of Antartic Marine Living Resourse, 1980, 19 ILM 841; Convention on the Regulation of Antartic Mineral Resources Activities, 1988; y, especialmente, Protocol on Environment Protection to the Antartic Treaty, artículo $7^{\circ}, 1991$. Actualmente, el Tratado del Ártico se encuentra en negociación y, de hecho, se está utilizando el Tratado del Antártico como base para su proyección, ya que, como se sabe, ambas zonas comparten características tanto climáticas como superficiarias. De esta forma, la inspiración y el espíritu resolutivo de este acuerdo está empapando las nuevas tratativas.

14 Cfr. Rio Declaration on Environment and Development 1992, principle 15: "In order to protect the environment, the precautionary approach shall be widely applied by states according to their capabilities. Where there are threats of serious or irreversible damage, lack of full scientific certainty shall not be used as a read on for postponing cost-effective measures to prevent environmental degradation".

15 Cfr. Duyck, "Drawing lessons for Artic governance...", op. cit. 
a contaminación y cambio climático, sino ser ampliado en lo relativo al turismo y la investigación. ${ }^{16}$ A título de ejemplo, Canadá aplica el principio precautorio como uno de los tres fundamentos estratégicos del derecho del mar, ${ }^{17}$ definiéndola como "erring on the side of caution", es decir, con el objeto de evitar la realización de acciones de riesgo y perjuicio. ${ }^{18}$

No obstante, la zona del Ártico contaba con algunas experiencias en antiguas convenciones y acuerdos aplicables a la protección de la flora y la fauna que aluden también, aunque con menor claridad, al principio precautorio. Un ejemplo de ello fue, en su momento, la Convención para la Protección y Preservación de Lobos Marinos en el Océano Pacífico Norte, de $1911 .^{19}$

\subsection{Distintas perspectivas de la Convención de las Naciones Unidas sobre el Derecho del Mar aplicables al cambio climático y a la protección de las regiones polares}

Según Boyle, la Convención de las Naciones Unidas sobre el Derecho del Mar de 1982 no alude, directamente, al cambio climático o a la contaminación del medio ambiente por la emisión de gases de efecto invernadero. ${ }^{20}$ Sin embargo, el autor afirma que la parte XII, al señalar la contaminación marina producida por buques y la protección de ecosistemas sensibles y especies en extinción, puede adecuarse perfectamente a la situación en las regiones del Ártico y la Antártida, especialmente por la aplicación genérica del artículo 192 de la Convención, que dispone la obligación, en cabeza de los Estados, de proteger y preservar el medio marino. En otras palabras, Boyle incluye tanto a ecosistemas frágiles como a hábitats especialmente

\footnotetext{
16 Idem.

17 Cfr. Hakon Hoel, Alf, "Best practices in ecosystems based oceans management in the Arctic", Report Series Number 129, 2009.

18 Cfr. Duyck, "Drawing lessons for Artic governance...", op. cit.

19 Conocido como Convention between the United States and Other Powers Providing for the Preservation and Protection of Fur Seals, celebrado por Estados Unidos, Reino Unido, Japón y Rusia, en http://pribilof.noaa.gov/documents/THE_FUR_SEAL_TREATY_OF_1911.pdf, consulta del 25 de julio de 2016.

20 Boyle, Alan, "Law of the sea perspectives on climate change", The International Journal of Marine and Coastal Law, 2012, 27, pp. 831-838.
} 
vulnerables, amenazados o en peligro de extinción, que, en definitiva, permite comprender en su totalidad a las zonas polares. ${ }^{21}$

Por otra parte, la contaminación del dióxido de carbono depositado en la atmósfera genera un riesgo inminente no solo para la biodiversidad marina presente en la columna de agua, sino también para todo el planeta, ya que, aplicando una lectura dinámica de la Convención y en particular del artículo 194, este aparece adaptable, pues hace referencia a las medidas necesarias para prevenir la contaminación marina de cualquier fuente. ${ }^{22}$ Así mismo, y considerando la norma señalada en conjunto con los artículos 207 (referido a la contaminación procedente de fuentes terrestres) a 212 (contaminación desde la atmósfera o a través de ella), advertimos una vinculación entre todas las fuentes de contaminación marina que permite ampliar nuestra idea.

Por último, resulta especialmente significativo como marco de protección de medio marino el artículo 234, aplicable a las zonas cubiertas de hielo, respecto del cual los Estados ribereños tienen derecho a dictar y hacer cumplir leyes y reglamentos — sin discriminación-, con el objeto de prevenir, reducir y controlar la contaminación del medio marino causada por buques en las zonas cubiertas de hielo, dentro de los límites de la zona económica exclusiva (ZEE), donde la especial severidad de las condiciones climáticas y la presencia de hielo durante la mayor parte del año creen obstrucciones o peligros excepcionales para la navegación, y la contaminación del medio marino pueda causar daños de importancia o alterarlo en forma irreversible. ${ }^{23}$ No obstante, las medidas adoptadas por

\section{Idem.}

22 En efecto, la norma dispone: “[...] Medidas para prevenir, reducir y controlar la contaminación del medio marino: 1. los Estados tomarán, individual o conjuntamente según proceda, todas las medidas compatibles con esta Convención que sean necesarias para prevenir, reducir y controlar la contaminación del medio marino procedente de cualquier fuente, utilizando a estos efectos los medios más viables de que dispongan y en la medida de sus posibilidades, y se esforzarán por armonizar sus políticas al respecto; 2 . los Estados tomarán todas las medidas necesarias para garantizar que las actividades bajo su jurisdicción o control se realicen de forma tal que no causen perjuicios por contaminación a otros Estados y su medio ambiente, y que la contaminación causada por incidentes o actividades bajo su jurisdicción o control no se extienda más allá de las zonas donde ejercen derechos de soberanía de conformidad con esta Convención [...]”.

23 Con fundamento en una entonces polémica legislación canadiense de los años setenta del siglo Xx, destinada a regular su jurisdicción sobre la navegación hasta una anchura de 100 millas para evitar la contaminación del mar por los buques, este artículo fue negociado por 
el Estado costero deben respetar, debidamente, la navegación, por lo que resulta necesario reconocer que se trata de un margen de discrecionalidad que no admite abusos y que, de presentarse la navegación, debe remitirse a las demás disposiciones de la Convención, particularmente las dispuestas en las partes II y III.

\section{E1 régimen de navegación en la Convención de las Naciones Unidas sobre el Derecho del Mar de 1982 y su aplicación al Northwest Passage}

\subsection{Consideraciones generales sobre el paso inocente en la Convención}

Se entiende por "paso inocente" a la navegación pacífica, rápida e ininterrumpida de un buque extranjero que no resulte perjudicial para la paz, el buen orden o la seguridad del Estado ribereño, ${ }^{24}$ el que puede regularla siempre que no imponga requisitos a estos buques con el objeto de denegar u obstaculizar el paso ni constituya, en la práctica, una discriminación. Tampoco permite establecer gravámenes por el solo hecho de realizarse el tránsito, excepto que se trate de un pago no discriminatorio ante de-

la propia Canadá, la entonces Unión Soviética y Estados Unidos, obteniendo aprobación por los gobiernos de estos países. Cfr. Symonides, "Problems and controversies...", op. cit., pp. 226-227. Aunque Symonides omite la información de la norma canadiense, se trata, con seguridad, de la Arctic Waters Pollution Prevention Act (AWPPA), adoptada en 1970 por aquel Estado. Cfr. Lalonde, Suzanne, "Evaluating Canada's position on the Northwest Passage in light of two possible sources of international protection", en Schofield, Clive, Lee, Seokwoo \& Kwon, Moon-Sang (eds.), The limits of maritime jurisdiction, Brill, Leiden, 2014, p. 577.

24 Cfr. artículos 19 y 20 de la Convención. Así mismo, para beneficiarse del paso inocente, este no puede ser utilizado por el buque para ingresar a las aguas interiores del Estado ribereño o salir de ellas, ni realizar escalas en radas o instalaciones portuarias. El carácter de paso rápido e ininterrumpido no impide que el buque pueda detenerse y fondear, siempre que ello sea producto de incidentes normales de la navegación, fuerza mayor, dificultad grave o que tenga el objeto de prestar auxilio a personas. Finalmente, las actividades contempladas en el artículo 19(2), a) a l), de la Convención son consideradas pasos perjudiciales, concluyendo que cualquier actividad no relacionada directamente con el paso impide calificarlo como 'inocente'. 
terminados servicios prestados (por ejemplo, el practicaje obligatorio por razones de seguridad marítima). ${ }^{25}$

Su antecedente más relevante fue el estudio, por parte de la CIJ, al resolver el primer caso sometido a su decisión: el asunto del Corfu Channel, en 1949. Allí la Corte afirmó que el paso era inocente, mientras no fuese perjudicial para la paz, el buen orden o la seguridad del Estado ribereño, ${ }^{26}$ aspecto que fue codificado en el artículo 17 de la Convención y que, actualmente, posee carácter consuetudinario. A pesar de lo dispuesto en el artículo 19, tendiente a establecer criterios objetivos para aclarar el significado de "paso inocente", ${ }^{27}$ la doctrina no acuerda en afirmar si las disposiciones referidas a este derecho (entre los artículos 14 y 23) pueden tener idéntica naturaleza, ya que poseen un carácter general amplio de interpretación discrecional a favor del Estado ribereño. ${ }^{28}$

Tal vez, una posible explicación de lo anterior encuentre fundamento en el resultado alcanzado en otra parte de la Convención: el régimen de estrechos internacionales, dado que muchos de ellos quedaban totalmente dentro del mar territorial de Estados ribereños, requiriendo que los negociadores consensuaran un sistema especial, ante la necesidad de asegurar un paso libre y sin obstáculos, especialmente por aquellos interesados en una rápida movilización de flotas navales, dentro de un contexto signado por la Guerra Fría. En otras palabras, en el régimen del mar territorial, el derecho de paso inocente es interpretado en beneficio del Estado ribereño, mientras que en los estrechos internacionales resulta favorable al Estado del pabellón del buque.

25 Cfr. artículos 21 a 26 de la Convención. El derecho de paso inocente no se aplica al sobrevuelo de las aeronaves, por tenerse en cuenta para este el Convenio sobre Aviación Civil Internacional de 1944. Igualmente, cabe señalar que en Chicago se firmaron otros acuerdos, como el Acuerdo de Tránsito y el Acuerdo de Transporte, que consagró las llamadas "libertades del aire", moderando la rigidez del Convenio de 1944 referido, de modo que la primera libertad acordada en el Acuerdo de Tránsito reconoce el derecho a sobrevuelo pacífico de aeronaves sobre el espacio aéreo de terceros Estados sin aterrizaje.

26 Cfr. Corfu Channel Case, Judgment of April $4^{\text {th }}, 1949$, ICJ Reports 1949, pp. 4 y ss.

27 La lista menciona actividades relacionadas con la soberanía, la integridad territorial, la independencia política, la defensa o seguridad del Estado ribereño, ejercicios navales, contaminación intencional, pesca, investigación, perturbación en comunicaciones, servicios e instalaciones, así como cualquier otra actividad que no tenga una relación directa con el paso.

28 Cfr. Wolf, Sarah, "Territorial sea”, en Wolfrum, The Max Planck encyclopedia..., op. cit., paras. 22-24. 
En definitiva, los buques extranjeros que ejerzan el derecho de paso inocente por el mar territorial deberán cumplir las leyes y reglamentos del Estado ribereño, así como las normas internacionales generalmente aceptadas relativas a la prevención de abordajes en el mar. Por su parte, el Estado ribereño puede intervenir o suspender temporalmente el paso, cuando lo requiera la seguridad en la navegación; puede designar vías marítimas y establecer dispositivos de separación del tráfico marítimo, en particular respecto de buques contenedores de hidrocarburos, buques de propulsión nuclear y aquellos que transporten sustancias o materiales nucleares u otros intrínsecamente peligrosos o nocivos, ${ }^{29}$ entre otras medidas para protección de su seguridad. ${ }^{30}$ El Estado ribereño tiene, en general, una amplia gama de facultades de ejecución contra buques extranjeros en caso de presentarse un paso no inocente, por ejemplo: detener, visitar e inspeccionar a los buques, pudiendo indicarles la salida del mar territorial, o bien dirigirlos a puerto para dar inicio al proceso judicial que corresponda.

Como corolario, el Estado ribereño ejerce su soberanía conforme la Convención y otras normas del derecho internacional aplicables, con la sola limitación de permitir el paso inocente de los buques que enarbolen el pabellón de otros Estados, los que deben navegar sin realizar actividades que requieran de la autorización del Estado costero o que sean contrarias a sus leyes. Ello significa, desde otro punto de vista, que este Estado puede, en principio sin limitación, regular y controlar prácticamente todas las actividades desarrolladas en el mar territorial, incluyendo el sobrevuelo.

\subsection{Los estrechos utilizados para la navegación internacional y el derecho de navegación. Su aplicación mutatis mutandis a las aguas archipelágicas}

Un estrecho es un curso de agua navegable que une dos zonas de mar, y su régimen presenta, en la práctica, dificultades de orden político y geográfico, que, asociadas a la libertad de navegación y comunicación, pueden generar aristas complejas, ${ }^{31}$ especialmente cuando el estrecho divide las costas de

\footnotetext{
29 Cfr. artículos 21(4) y 22(1) y (2) de la Convención.

30 Cfr. artículo 25 de la Convención.

31 Cfr. Caminos, Hugo, "The legal régime of straits in the 1982 United Nations Convention on the Law of the Sea", Recueil des Cours, 1987, 205, pp. 19 y ss.
} 
dos Estados. ${ }^{32}$ Su definición legal se basa en dos elementos: una condición geográfica referida a dos grandes masas de agua conectadas entre sí; y el carácter funcional de ser utilizado para la navegación internacional. Su régimen es un compromiso de gran importancia tanto para los Estados que desean ejercer su paso como para aquellos interesados en supervisar y regular el tráfico de estas aguas. ${ }^{33}$

Históricamente, su antecedente se encuentra en los trabajos del Institut de Droit International elaborados entre 1894 y 1912; en el debate en la Conferencia de La Haya de 1907; y en las tareas de la International Law Association (ILA). ${ }^{34}$ Respecto del paso de buques de guerra en tiempos de paz, la CIJ tuvo ocasión de analizarlo en el caso del Corfu Channel, reconociendo a aquellos el derecho de paso, ya que ese estrecho unía dos partes de alta mar y se utilizaba para la navegación internacional, a pesar de su carácter secundario respecto de otras vías. ${ }^{35}$

Posteriormente, el derecho consuetudinario en materia de estrechos fue codificado y se reflejó en el artículo 16, inciso 4, de la Convención sobre Mar Territorial y la Zona Contigua, de 1958, estableciendo los requisitos por cumplirse para considerar a un curso de agua como un estrecho

\footnotetext{
32 Para una primera aproximación al concepto de 'estrechos utilizados para la navegación internacional' (o estrechos internacionales), nos remitimos a la sentencia de la CIJ en el Corfu Channel ya referido. En el caso, Albania había sostenido que el estrecho no era una vía marítima internacional por tratarse de una vía secundaria que se empleaba casi con exclusividad para el comercio local entre Jacaranda y Corfú. La CIJ desestimó estos argumentos y destacó la "situación geográfica de estrecho" al comunicar dos zonas de alta mar y que este era utilizado para la navegación internacional. En el caso de la República Argentina, reviste especial interés el estatus jurídico del estrecho de Magallanes. Para profundizar esta cuestión en particular, sugerimos Caminos, “The legal régime of straits...”, ibid., pp. 221 y ss.

33 Cfr. Lapidoth, Ruth, "Straits, international", en Wolfrum, The Max Planck encyclopedia..., op. cit., para. 1.

34 Cfr. Caminos, "The legal régime of straits...”, op. cit., pp. 28-29.

35 Cfr. Corfu Channel Case, Judgment of April 4 ${ }^{\text {th }}, 1949$, ICJ Reports 1949, pp. 4 y ss. Para profundizar sobre la libre navegación de buques de guerra, incluyendo el régimen de estrechos, ver Godio, Leopoldo M. A. \& Rosenthal, Julián M., "La libre navegación de los buques de guerra en el mar territorial y su regulación a partir de la Convención de las Naciones Unidas sobre el Derecho del Mar de 1982", Ars Iuris Salmanticensis, 2015, 3, (2), pp. 109-131.
} 
internacional. Sin embargo, algunos autores interpretan que esta norma constituyó, en parte, un desarrollo progresivo. ${ }^{36}$

No obstante, el régimen de estrechos generó arduos debates durante la III Conferencia y, finalmente, la Convención estableció en su parte III las disposiciones jurídicas aplicables para los estrechos destinados a la navegación internacional. Su articulado concilia, de modo equilibrado, los intereses de potencias mercantes y militares, exigiendo una fluidez en el tránsito internacional y las mayores opciones de operatividad para sus fuerzas navales; ${ }^{37}$ y los del Estado costero del estrecho, preocupados, fundamentalmente, por la seguridad estratégica defensiva de sus costas y la protección ambiental. ${ }^{38}$

Al respecto, Scovazzi asevera que el régimen de estrechos internacionales establecido en la Convención fue un punto crucial del package deal. ${ }^{39}$ En ese sentido, solo los estrechos utilizados para la navegación internacional son regulados por la Convención, en los que rige un derecho de paso establecido por la misma parte III, ${ }^{40}$ encontrándose los Estados costeros obligados a no obstaculizarlo e informar todo peligro que amenace su navegación o sobrevuelo. Así mismo, si un estrecho utilizado para la navegación internacional une dos zonas de alta mar, o el alta mar y una ZEE, o dos ZEE de dos Estados distintos, se aplica al respecto el derecho de paso para la navegación y el sobrevuelo previsto en la sección II de la parte III de la Convención. ${ }^{41}$

A pesar de su aparente similitud con el régimen de paso inocente previsto para el mar territorial, este resulta más favorable a los Estados en ejercicio del derecho en tránsito, puesto que se aplica también a las aeronaves $^{42}$ e incluye, discutiblemente, el derecho de paso sumergido para

\footnotetext{
36 Cfr. Lapidoth, "Straits, international", op. cit., para. 4.

37 Cfr. Scovazzi, Tullio, "Evolution of international law of the sea", Recueil des Cours, 2000, 286, pp. 173-187.

38 Cfr. Treves, Tullio, "Codification du droit international et pratique des États dans le droit de la mer”, Recueil des Cours, 1990, 223, pp. 9 y ss. Para un análisis del tema en particular, ver Fornari, Matteo, Il regime giuridico degli stretti utilizzati per la navigazione internazionale, Giuffrè Editore, Milano, 2010.

39 Cfr. Scovazzi, "Evolution of international law of the sea", op. cit., p. 168.

40 Cfr. artículo 37 de la Convención.

41 Cfr. artículos 38 y 39 de la Convención.

42 Cfr. artículo 38 de la Convención.
} 
los submarinos. ${ }^{43}$ Por otra parte, el Estado costero puede dictar leyes y reglamentos en materia de seguridad de la navegación; prevención, reducción y control de la contaminación; prohibición de pesca; y embarco o desembarco de productos, monedas o personas. ${ }^{44}$ En definitiva, los estrechos constituyen un régimen especial en materia de navegación, que permite, con requisitos, establecer disposiciones en materia de conservación de recursos vivos y no vivos, incluso en estrechos que constituyan una frontera entre dos Estados. ${ }^{45}$

Finalmente, en aguas archipelágicas, los deberes de los buques y aeronaves durante su paso, incluyendo las actividades de investigación y estudio, así como los deberes del Estado archipelágico y las disposiciones referidas al tránsito por las vías marítimas archipelágicas, remiten a los artículos 39, 40, 42 y 44 de la Convención, que se aplican, mutatis mutandis, al paso por las vías marítimas archipelágicas. ${ }^{46}$ En lo referido al paso, el artículo 53(3), (4) y (12) aporta algunas aclaraciones al concepto de "paso por las vías marítimas archipelágicas", entendiéndole como:

[...] el ejercicio, de conformidad con esta Convención, de los derechos de navegación y de sobrevuelo en el modo normal, exclusivamente para los fines de tránsito ininterrumpido, rápido y sin trabas entre una parte de la alta mar o de una zona económica exclusiva y otra parte de la alta mar o de una zona económica exclusiva.

4. Tales vías marítimas y rutas aéreas atravesarán las aguas archipelágicas y el mar territorial adyacente e incluirán todas las rutas normales de paso utilizadas como tales en la navegación o sobrevuelo internacionales

43 Cfr. Wolf, "Territorial sea”, op. cit., para. 26.

44 Cfr. artículo 42(1) de la Convención.

45 Cabe destacar que el régimen, para algunos Estados como Estados Unidos, reviste carácter de norma consuetudinaria — cfr. UN, LoS Bulletin, 1988, (12), p. 18-, o bien se trata de un régimen que no posee aceptación general en virtud de distintas declaraciones realizadas, como Irán (que no es parte, con relación al estrecho de Ormuz), en los siguientes términos: "[...] certain of its provisions are merely the product of quid-pro-quo which do not necessarily purport to codify the existing customs or established usage (practice) regarded as having an obligatory character' y que 'the right of transit passage through straits used for international navigation (part III, section 2, article 38)". En http:/ / www.un.org/ depts/los/convention_agreements/convention_declarations.htm\#IranUponsignature, consulta del 25 de julio de 2016.

46 Cfr. artículo 54 de la Convención. 
a través de las aguas archipelágicas o sobre ellas y dentro de tales rutas, en lo que se refiere a los buques, todos los canales normales de navegación $[\ldots]$;

12. Si un Estado archipelágico no designare vías marítimas o rutas aéreas, el derecho de paso por vías marítimas archipelágicas podrá ser ejercido a través de las rutas utilizadas normalmente para la navegación internacional. ${ }^{47}$

Lo anterior nos lleva a preguntarnos si al Northwest Passage se le deben aplicar las disposiciones referidas a la parte III, referida a los estrechos internacionales; las establecidas en la parte IV, reconocidas para las aguas archipelágicas; o ninguna de ellas, recurriendo a la Convención y las disposiciones específicas que se presentan en las particularidades del caso.

\section{E1 caso particular del Northwest Passage. El debate entre Estados Unidos y Canadá}

Los efectos del cambio climático, evidentes en las regiones polares y, principalmente, en el Ártico, advierten sobre las oportunidades que brindan los mares de agua-hielo como oportunidad para la navegación y el comercio, empleando sus rutas para efectuar y perfeccionar este intercambio. Sin lugar a dudas, una de las vías más conocidas es el paso del Noroeste, también llamado Northwest Passage, que se extiende a través del archipiélago ártico de Canadá, ${ }^{48}$ con un gran potencial como espacio estratégico que no solo acortaría y abarataría los costos marítimos en el transporte entre Europa y la costa este de los Estados Unidos, sino que también comunicaría esta con el continente asiático. ${ }^{49}$

En este contexto es que Estados Unidos, Rusia, Japón y la Unión Europea intentan obtener, permanentemente, beneficios para maximizar

\footnotetext{
47 Los destacados en cursiva no pertenecen al texto original.

48 Handl, Günther, "Northwest Passage (Canadian-American controversy)", en Wolfrum, The Max Planck, encyclopedia..., op. cit.

49 Griffiths, Frederick, Politics of the Northwest Passage, McGill-Queen's University Press, Kingston, 1987, pp. 1-3. Según Handl, distintos informes de la ONU reconocen que el tráfico comercial a través del paso estará restringido solo estacionalmente en un futuro cercano, aunque las condiciones ambientales, de por sí desafiantes y controvertidas, harán del comercio en las regiones polares algo incierto. Cfr. Handl, "Northwest Passage...", ibid.
} 
sus intereses y, en consecuencia, advierten las distintas alternativas que presentan las regiones polares..$^{50}$ Así fue como realmente han tenido inicio todas las controversias en torno al Northwest Passage, y por esta misma razón es que las disputas llevan años de duración y han contado con la participación de muchos actores internacionales para proponer una solución que contemple sus intereses.

El inicio de la tesis canadiense se sitúa en 1969 cuando un buque tanque intentó atravesar el archipiélago Ártico de Canadá para comprobar la posibilidad de transportar petróleo desde Alaska hasta el noroeste de Estados Unidos y tal vez Europa. El Estado canadiense lo consideró como una intromisión a su territorio que afectó su soberanía, aunque este aspecto es cuestionado por parte de la doctrina, que desconoce el dominio canadiense en estas extensiones. En el año 1970 y motivado por este incidente, Canadá extendió su mar territorial a 12 millas y dispuso una zona de conservación de hasta 100 millas, con el objeto de administrar el espacio con base en una "soberanía funcional y cuidado" de zonas delicadas. Fue en 1973 cuando el gobierno canadiense calificó el paso como aguas interiores, negando reconocerle el carácter de estrecho internacional, y, así, intentar obtener pleno dominio sobre las aguas del archipiélago Ártico. Las controversias comienzan a proliferar cuando, en 1985, Canadá insistió en requerir a Estados Unidos una autorización previa para permitir el paso de un buque rompehielos. Estados Unidos se negó rotundamente a esto, alegando que el Northwest Passage reviste carácter de estrecho internacional. ${ }^{51}$

Estas disputas convergieron, en 1988, con un acuerdo de cooperación en el Ártico referido al tránsito en el paso del Noroeste que impide otorgarle efecto jurídico, en relación con las respectivas posiciones de los Estados entre ellos ni tampoco respecto de terceros, a toda navegación realizada por buques rompehielos norteamericanos. ${ }^{52}$ En otras palabras, ambos Estados reafirmaron sus posiciones en la controversias reduciendo

50 Cfr. Franckx, Erik, Maritime claims in the Artic: Canadian and Russian perspectives, Martinus Nijhoff, Dordrecht, 1993.

51 Handl, "Northwest Passage...", op. cit.

52 Canada Treaty Information, Agreement between the Government of the United States of America on Artic Cooperation, E 101701 - CTS, 1988, № 29, en www.treaty-accord. gc.ca, consulta del 25 de julio de 2016. 
la cuestión, según Symonides, a si el Northwest Passage puede ser reconocido como un estrecho utilizado para la navegación internacional. ${ }^{53}$

Desde aquel momento pocos rompehielos cumplieron con la formalidad de la notificación y autorización previa. El fundamento es ideológico: Estados Unidos continúa afirmando que no solo la ruta en cuestión presenta carácter de libre navegación, sino también todas las rutas conformadas por las islas del Ártico, negando a estas el estatus de aguas interiores canadienses. ${ }^{54}$

Sobre este punto, Symonides se pregunta si las condiciones geográficas que conecten las aguas de los estrechos destinados a la navegación internacional resultan suficientes o bien si estas deben complementarse con un uso funcional para el transporte marítimo. ${ }^{55}$ Por evidentes razones, Canadá alega que esta condición no es cumplida en el caso del Northwest Passage. Sin embargo, en el supuesto de reducir el debate a una calificación de estrecho internacional que la prudencia no permite asegurar de modo concluyente, adelantamos que la Convención de las Naciones Unidas sobre el Derecho del Mar no contiene disposiciones de particular y especial aplicación al caso en estudio, aunque es posible razonar que de la propia naturaleza que poseen estrechos internacionales destinados a la navegación, regulados en la parte III de la Convención, debe prevalecer el tránsito del buque por sobre las restricciones del Estado costero.

Igualmente, este ensayo de criterio que hemos adelantado revela la inconsistencia de los argumentos canadienses al exigirse una 'práctica' que habilite la funcionalidad cuando, por otro lado, este Estado interpreta el acuerdo de 1988 celebrado con Estados Unidos — ya señalado- como una limitación en ese sentido.

En razón de lo expuesto, si se considerase al Northwest Passage como un estrecho, no quedan muchos fundamentos para la oposición canadiense, ni siquiera ante buques o aeronaves de guerra extranjeros,

\footnotetext{
53 Cfr. Symonides, "Problems and controversies...", op. cit., p. 238.

54 En consecuencia, afirma Handl, no se presenta evidencia que permita concluir la existencia de títulos canadienses en relación con el paso del Noroeste y sus aguas circundantes, concluyendo que su reclamo no tiene fundamento de acuerdo con el derecho internacional, especialmente si se consideran los problemas de delimitación que presenta en el golfo de Amundsen y el estrecho de McClure. Cfr. Handl, "Northwest Passage...", op. cit.

55 Cfr. Symonides, "Problems and controversies...", op. cit., pp. 238-239.
} 
excepto que se encuadre dentro de las excepciones a la navegación, de carácter restrictivo, que habilita la parte III de la Convención. ${ }^{56}$

Por otra parte, corresponde rechazar al Northwest Passage como una vía de navegación conformada por un conjunto de islas ${ }^{57}$ que, a su vez, disponga el establecimiento de vías marítimas archipelágicas, ya que, en este supuesto que remite a un paso sin restricciones, la navegación y sobrevuelo debe realizarse del modo 'normal'. Este resultado para el caso del Northwest Passage es la que propone Vincent Cogliati-Bantz, en criterio que compartimos, con fundamento en las negociaciones e interpretaciones formuladas en ocasión de la III Conferencia. ${ }^{58}$

En definitiva, independientemente de prevalecer el criterio de una tesis u otra, lo único que parece indiscutible es que el cambio climático permitiría una gradual 'normalización' del tránsito en el Northwest Passage, que, sostenemos, con el tiempo favorecería la interpretación de los intereses y la posición del gobierno de Estados Unidos tendiente a asegurar la navegación por estas aguas.

Lo anterior adquiere aún más fundamento al considerar la evaluación de la postura canadiense, sintetizada por Suzanne Lalonde, quien afirma que los derechos canadienses sobre el control del Northwest Passage se encuentran desafiados ante el creciente acceso en la región del Ártico y el aumento del transporte marítimo, ${ }^{59}$ ensayando propuestas de protección a partir de dos fundamentos: 1) la aplicación del artículo 234 de la Convención ya referido; o 2) considerar al Northwest Passage como una "zona marina particularmente sensible" (particularly sensitive sea area, PSSA). ${ }^{60}$

56 Cfr. Godio \& Rosenthal, "La libre navegación de los buques de guerra...”, op. cit., pp. 109-131.

57 Cfr. artículo 121 de la Convención. Sobre el régimen de islas y su interpretación por tribunales internacionales, ver Godio, Leopoldo M. A., "La evolución del régimen jurídico de las islas en el derecho internacional contemporáneo y su interpretación en la controversia entre Nicaragua y Colombia", en Alice, Mauricio et al., Contribuciones al estudio del derecho internacional, Consejo Argentino para las Relaciones Internacionales (CARI), Buenos Aires, 2015, pp. 157-182.

58 Cfr. Cogliati-Bantz, Vincent P., "Archipelagic States and the new law of the sea", en Del Castillo, Law of the sea..., op. cit., p. 304.

59 Cfr. Lalonde, "Evaluating Canada's position...”, op. cit., pp. 575-588.

60 Se trata de áreas que, debido a su reconocida importancia ecológica, socioeconómica o científica, pueden ser vulnerables a los daños causados por las actividades marítimas internacionales. Los criterios para su identificación y requisitos de designación están contenidos 
Actualmente, el Northwest Passage es utilizado para la navegación, aunque solo parcial y esporádicamente durante el verano, y presenta, respecto de la Northern Sea Route, una diferencia relevante, ya que esta última es utilizada durante todo el año por buques de la Federación de Rusia, que cuenta con la más importante flota de rompehielos del mundo y mantiene, desde 1991, esta ruta abierta para la navegación internacional. ${ }^{61}$

Llegados a este punto, ¿constituye la Convención y sus disposiciones un instrumento completo para su regulación, o bien es necesario recurrir a un instrumento específico atento a las particularidades que presenta la zona del Northwest Passage? En una interpretación preliminar, puede reconocerse la aplicación de muchas instituciones de la Convención, tales como aguas interiores, mar territorial, estrechos utilizados para la navegación internacional, ZEE, alta mar y la protección del medio marino, que resultan prima facie suficientes. Sin embargo, en tiempos recientes han aparecido nuevas regulaciones: en junio de 2010, Canadá dispuso la vigencia obligatoria del Northern Canada Vessel Traffic Services Zone Regulations (conocido como Nordreg, por su acrónimo en inglés), un sistema de información de entrada y navegación, aplicable a los buques que ingresen en aguas situadas al norte de Canadá. ${ }^{62}$

La obligatoriedad de la norma mereció el rechazo de gran parte de los armadores, además de los gobiernos de Estados Unidos, la Unión

en la directriz de la OMI, revisada en 2005, A.982(24) Revised guidelines for the identification and designation of particularly sensitive sea areas (PSSAS), comprendiendo a los ecosistemas únicos o raros; la biodiversidad y su vulnerabilidad; criterios sociales, culturales y económicos, como la importancia de la zona para la recreación o el turismo; y criterios científicos o históricos, entre otros. Una vez que se le designa como PSSA, es posible adoptar medidas específicas de control para las actividades marítimas, como, por ejemplo, establecer criterios de separación del tráfico o aplicar requisitos más estrictos de carga y descarga en buques. Actualmente, existen 16 áreas determinadas como "particularmente sensibles" por la OMI. En http://www.imo.org/es/OurWork/Environment/PSSAs/Paginas/Default. aspx, consulta del 25 de julio de 2016.

61 Cfr. Symonides, "Problems and controversies...", op. cit., p. 226.

62 En http://laws-lois.justice.gc.ca/eng/regulations/SOR-2010-127/, consulta del 25 de julio de 2016. Sus disposiciones, con sanciones que prevén multas de hasta 100000 dólares estadounidenses, son aplicables a: 1) buques de más de 300 toneladas; 2) remolcadores que, sumando el tonelaje del buque remolcado, iguale o supere las 500 toneladas; y 3) los buques que transporten cargas peligrosas o contaminantes, incluyendo a sus remolcadores. Para un análisis de la norma, ver Kraska, James, "The Northern Canada Vessel Traffic Services Zone Regulations (Nordreg) and the law of the sea", International Journal of Marine and Coastal Law, 2015, 30, pp. 225-254. 
Europea y Singapur, al interpretar que se trata de una violación al principio de la libertad de navegación que no guarda relación con los derechos de soberanía reconocidos en la ZEE. ${ }^{63}$ Sin embargo, no resultaría difícil justificar la postura canadiense aplicando los grandes principios ambientales y las disposiciones de protección del medio marino, especialmente si se consideran los peligros adicionales y las condiciones que influyen en la navegación por estas aguas.

Symonides afirma que existe una gran cantidad de normas, códigos, directrices y recomendaciones vinculantes que garantizan la seguridad de los buques, su tripulación y sus pasajeros. No obstante, manifiesta que estas, a pesar de tener un impacto positivo, no resultan suficientes para la navegación en el Ártico. El único documento previsto expresamente no posee carácter vinculante y fue adoptado por dos órganos de la Organización Marítima Internacional (OMI): el Comité de Seguridad Marítima y el Comité de Protección del Medio Marino, en octubre y diciembre de 2002, respectivamente, que reconocen las exigencias adicionales que requiere el ambiente polar, debido a las condiciones climáticas de las aguas árticas cubiertas de hielo, para los buques y embarcaciones a partir de las 500 toneladas, clasificados según las condiciones de navegación y tipo de buque. ${ }^{64}$

Realizando un paralelismo, cabe señalar que los miembros consultivos pertenecientes al Sistema del Tratado Antártico solicitaron a la OMI, en 2004, la elaboración de directrices para los buques que operen en las aguas al sur del paralelo 60 Sur, ${ }^{65}$ adoptadas, finalmente, en 2009 y en vigor desde el $1^{\circ}$ de enero de $2011 .{ }^{66}$ No obstante lo anterior, cabe señalar que en febrero de 2010 la OMI comenzó la elaboración de un proyecto de código para la navegación en las aguas del Ártico y la Antártida. ${ }^{67}$

\footnotetext{
63 Cfr. Symonides, "Problems and controversies...", op. cit., p. 230.

64 Las directrices se dividen en cuatro partes: a) construcción de buques, b) equipo, c) operación y d) protección del medio marino y daños control. Ibid., pp. 231-232.

65 Ver: Aтсм Decision 4, "Guidelines for ships operating in Arctic and Antarctic icecovered waters", АтCM XXVII (24 May-4 June 2004).

66 A 26/Res.1024, adopted on 2 December 2009, Agenda item 10, 18 January 2010, en https://www.govmin.gl/images/stories/petroleum/IMO_A.102426_Guidelines_for_ ships_operating_in_polar_waters.pdf, consulta del 25 de julio de 2016.

67 Symonides, "Problems and controversies...", op. cit., p. 233.
} 


\section{Conclusión}

El derretimiento del océano Ártico presenta una importante oportunidad para el desarrollo del transporte internacional a través de la conexión de las rutas marítimas, permitiendo así reducir los costos comerciales y ambientales como consecuencia del transporte marítimo entre Europa y Asia, incluyendo a los Estados de América del Norte. ${ }^{68}$

Lo anterior no significa, per se, una consecuencia negativa desde el inicio, ya que posibilita un importante ahorro de combustible y una reducción en las emisiones de los gases que provocan el efecto invernadero, ${ }^{69}$ además de interesantes perspectivas para el desarrollo de la actividad turística y el proporcionar una fuente de acceso de agua potable, al menos teóricamente. ${ }^{70}$

Sin embargo, implementar estas actividades implica peligros para el medio marino al elevarse los niveles de ruido, afectar el hábitat de los mamíferos marinos e irrumpir a la población allí asentada. ${ }^{71}$ En otras palabras, el inicio de cualquier acción merece ser estudiada seriamente a fin de no afectar intereses y otros bienes tutelados. Lo importante por considerar, más allá del debate sobre el régimen aplicable a la navegación o conservación del Ártico y el paso del Noroeste como un 'área marina sensible', es el rol de los Estados interesados en estos espacios, a fin de no solo promover los instrumentos internacionales necesarios, por los mecanismos diplomáticos o foros especializados adecuados, como, por ejemplo, la OMI, sino también su cumplimiento.

Este último aspecto requiere, de acuerdo con la práctica internacional, de un sistema de solución de controversias realista, como el que se encuentra presente en la Convención en su parte XV. Sin embargo, cabe señalar que el artículo 298(1)(b) exceptúa del sistema de arreglo las controversias

\footnotetext{
68 Ibid., pp. 225-226.

69 Para una concientización sobre el calentamiento global y sus efectos, ver Gore, Una verdad incómoda..., op. cit. El autor afirma que "[...] somos testigos de un choque sin precedentes, gigantesco, entre nuestra civilización y la Tierra”. Ibid., p. 214.

70 Cfr. Symonides, "Problems and controversies..., op. cit., p. 225.

71 Un ejemplo de las dificultades, peligros y seriedad en la región puede encontrarse en Aveling, Nick, "Fuel tanker grounded in Northwest Passage", National Post, September 2, 2010, en http:/ / news.nationalpost.com/news/canada/ fuel-tanker-grounded-in-northwestpassage, consulta del 25 de julio de 2016.
} 
referidas a aguas históricas ${ }^{72}$ y las disputas de soberanía, razón por la cual resultaría necesario avanzar en un acuerdo bilateral o regional específico, máxime si Estados Unidos continúa sin ratificar la Convención.

Finalmente, corresponde adoptar una conclusión sobre qué parte de la Convención debe aplicarse al Northwest Passage, tal como lo hemos planteado al comienzo de nuestro trabajo. El desarrollo de la controversia entre Canadá y Estados Unidos se dirige, a pesar de los tratados bilaterales y sus declaraciones, a una práctica que favorece la postura de este último Estado, que pretende emplear las disposiciones previstas para los estrechos internacionales y las aguas archipelágicas, en el caso de que corresponda este criterio final para este paso en particular.

En ese sentido, cabe recordar que no es posible exigir a la Convención requisitos no previstos y que en los estrechos internacionales destinados a la navegación deben prevalecer las disposiciones del tránsito de buques por sobre las restricciones del Estado costero, en este caso, Canadá. Su fundamento se refuerza, incluso, gracias a la práctica efectuada por los Estados como consecuencia del cambio climático y que nos permite concluir una inevitable aplicación, tarde o temprano, fundada en la costumbre internacional. No obstante, el derecho internacional requiere prudencia y, por su carácter dinámico, es posible la aparición de opciones de origen diplomático que eviten un desenlace a favor del paso y que contemple en una solución híbrida los intereses de ambos Estados, reconociendo la navegación y, simultáneamente, disponiendo pautas de protección propias de un área marina sensible.

\section{Referencias}

Abello Galvis, Ricardo, "Eaux et baies historiques en droit international", Estudios Socio-Jurídicos, 2003, 5, (1), pp. 33-76.

Boyle, Alan, "Law of the sea perspectives on climate change", The International Journal of Marine and Coastal Law, 2012, 27, pp. 831-838.

\footnotetext{
$72 \mathrm{Al}$ respecto, cabe señalar que la Convención de 1982 no posee definición del concepto 'aguas históricas' y la única referencia a la idea se encuentra en su artículo 10(6), concerniente a las bahías que revisten esta característica, como podría ser, por ejemplo, el golfo de Fonseca. Para un análisis particular en este mismo sentido, ver Abello Galvis, Ricardo, "Eaux et baies historiques en droit international", Estudios Socio-Jurídicos, 2003, 5, (1), pp. 33-76.
} 
Byers, Michael, "Artic region: specific geographic issues", en Wolfrum, Rüdiger (ed.), The Max Planck encyclopedia of public international law, Oxford University Press, Oxford, 2012.

Caminos, Hugo, "The legal régime of straits in the 1982 United Nations Convention on the Law of the Sea", Recueil des Cours, 1987, 205, pp. 9-245.

Cogliati-Bantz, Vincent P., "Archipelagic States and the new law of the sea", en Del Castillo, Lilian (ed.), Law of the sea, from Grotius to the International Tribunal for the Law of the Sea. Liber amicorum judge Hugo Caminos, Brill, Leiden, 2015, pp. 299-317.

Durán Cotrina, Patricia, "La economía del cambio climático", Revista Peruana de Derecho Internacional, septiembre-diciembre 2011, LXI, (144), pp. 179-196.

Duyck, Sebastien, "Drawing lessons for Artic governance from the Antartic Treaty System", en Alfredsson, Gudmundur, Koivurova, Timo \& Hossain, Kamrul (eds.), The yearbook of Polar law. Vol. 3, Brill, Leiden, 2011.

Fernández Egea, Rosa M., "El cambio climático y la sostenibilidad de los recursos pesqueros del mar del Norte: la controversia comercial entre la Unión Europea y las islas Feroe", Revista del Instituto Español de Estudios Estratégicos (IEEE), 2014, 4.

Fornari, Matteo, Il regime giuridico degli stretti utilizzati per la navigazione internazionale, Giuffrè Editore, Milano, 2010.

Franckx, Erik, Maritime claims in the Artic: Canadian and Russian perspectives, Martinus Nijhoff, Dordrecht, 1993.

Godio, Leopoldo M. A. \& Rosenthal, Julián M., "La libre navegación de los buques de guerra en el mar territorial y su regulación a partir de la Convención de las Naciones Unidas sobre el Derecho del Mar de 1982", Ars Iuris Salmanticensis, 2015, 3, (2), pp. 109-131.

Godio, Leopoldo M. A., "La evolución del régimen jurídico de las islas en el derecho internacional contemporáneo y su interpretación en la controversia entre Nicaragua y Colombia", en Alice, Mauricio et al., Contribuciones al estudio del derecho internacional, Consejo Argentino para las Relaciones Internacionales (CARI), Buenos Aires, 2015, pp. 157-182.

González Napolitano, Silvina S., "Los desastres naturales y sus efectos en islas y Estados insulares: posibles soluciones jurídicas”, en González Napolitano, Silvina Sandra (dir.), Respuestas del derecho internacional a 
desastres y otras consecuencias de fenómenos naturales, SGN Editora, Avellaneda, 2015, pp. 97-110.

Gore, Albert, Una verdad incómoda: la crisis planetaria del calentamiento globaly cómo afrontarla, Gedisa, Barcelona, 2007.

Griffiths, Frederick, Politics of the Northwest Passage, McGill-Queen's University Press, Kingston, 1987.

Hakon Hoel, Alf, "Best practices in ecosystems based oceans management in the Arctic", Report Series Number 129, 2009.

Handl, Günther, "Northwest Passage (Canadian-American controversy)", en Wolfrum, Rüdiger (ed.), The Max Planck encyclopedia of public international law, Oxford University Press, Oxford, 2012.

Kraska, James, "The Northern Canada Vessel Traffic Services Zone Regulations (Nordreg) and the law of the sea", International Journal of Marine and Coastal Law, 2015, 30, pp. 225-254.

Lalonde, Suzanne, "Evaluating Canada's position on the Northwest Passage in light of two possible sources of international protection", en Schofield, Clive, Lee, Seokwoo \& Kwon, Moon-Sang (eds.), The limits of maritime jurisdiction, Brill, Leiden, 2014, pp. 575-588.

Lapidoth, Ruth, "Straits, international”, en Wolfrum, Rüdiger (ed.), The Max Planck encyclopedia of public international law, Oxford University Press, Oxford, 2012.

Martínez Vargas, Juan R., Vega Barbosa, Giovanny et al., Tratado de Derecho del Mar, Tirant lo Blanch, Valencia, 2016.

Scovazzi, Tullio, "Evolution of international law of the sea", Recueil des Cours, 2000, 286, pp. 39-243.

Symonides, Janusz, "Problems and controversies concerning freedom of navigation in the Arctic", en Del Castillo, Lilian (ed.), Law of the sea, from Grotius to the International Tribunal for the Law of the Sea. Liber amicorum judge Hugo Caminos, Brill, Leiden, 2015, pp. 225-242.

Treves, Tullio "Codification du droit international et pratique des États dans le droit de la mer", Recueil des Cours, 1990, 223, pp. 9-302.

Wolf, Sarah, "Territorial sea”, en Wolfrum, Rüdiger (ed.), The Max Planck encyclopedia of public international law, Oxford University Press, Oxford, 2012. 